
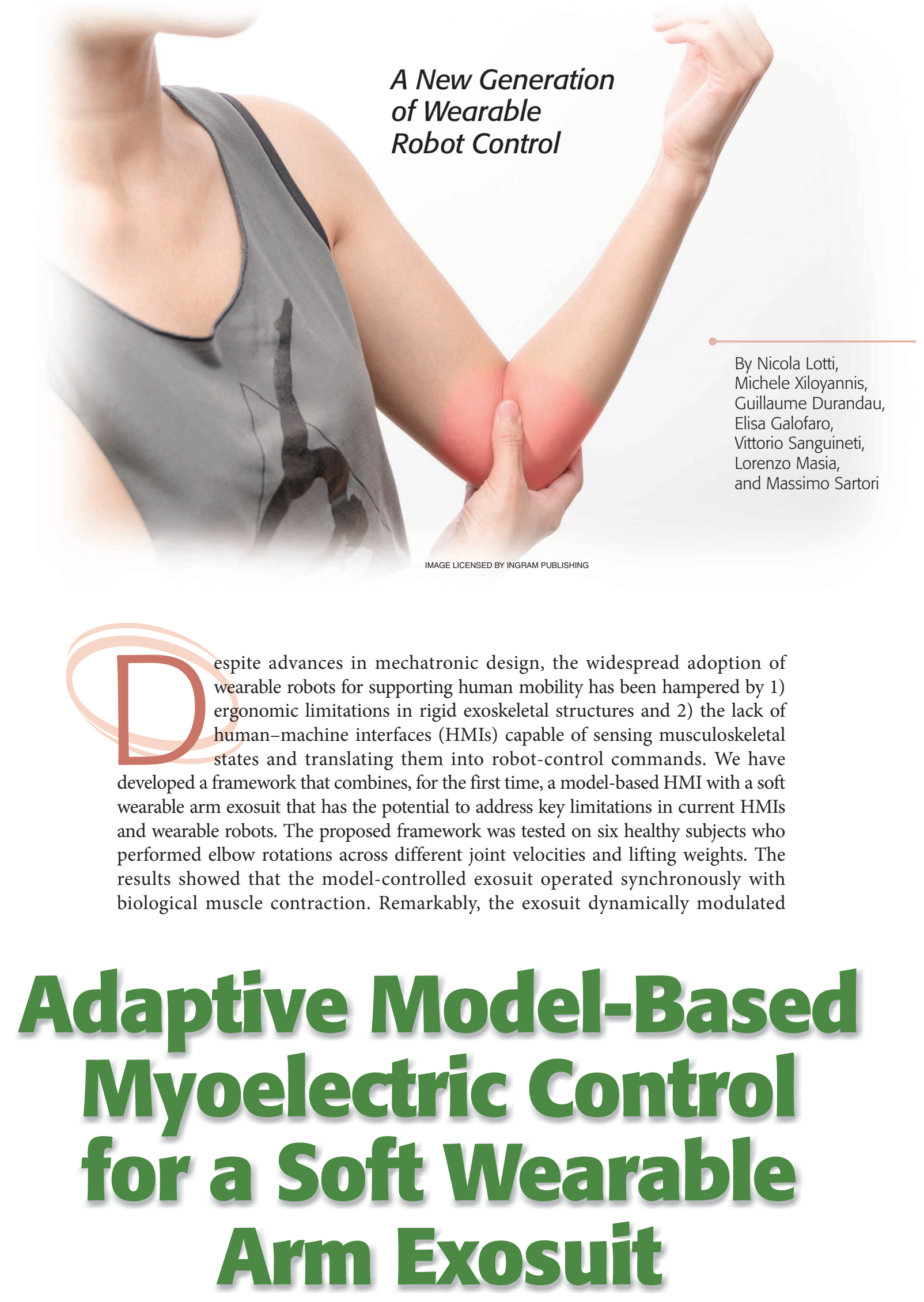

Digital Object Identifier 10.1109/MRA.2019.2955669

Date of current version: 20 January 2020 
mechanical assistance across all investigated loads, thereby displaying adaptive behavior.

As a result, the exosuit's intrinsic dynamics and the external mechanical loads appeared to be transparent to the individuals' musculoskeletal systems. This was reflected by the fact that, with exosuit assistance, the muscle electromyograms (EMGs) and resulting forces varied within comparable ranges across all investigated rotational velocities and loads; that is, the external load effect on the muscle function was minimized. However, when the exosuit assistance was off, the muscle activity and forces increased significantly across velocities and weights. The ability to seamlessly combine musculoskeletal-force estimators with

The quest for coexistence

\section{between humans and}

machines has pushed

$$
\text { scientific boundaries }
$$

beyond industrial

automation toward the

$$
\text { area of wearable robotics. }
$$
wearable soft mechatronics opens new avenues for assisting human movement in healthy and impaired individuals.

\section{Wearable Robot Limitations}

The quest for coexistence between humans and machines has pushed scientific boundaries beyond industrial automation toward the area of wearable robotics. With regard to motor rehabilitation and augmentation, a wealth of wearable robots has been designed for providing movement assistance. In this context, the aim is to compensate for reduced and missing voluntary activity via controlled forces that support taskbased motor activities. Yet technological constraints hamper the adoption of wearable robots and their widespread acceptance by final users: limitations in ergonomics, motorintention prediction, and control robustness represent major open challenges.

Existing wearable robots (that is, active orthoses and exoskeletons) interact with their users while providing little information about the effects induced on the musculoskeletal system. It is unknown how the human body responds to different HMI types and how wearable robots should operate to best enhance movements [1]. In this context, HMIs for active orthoses and exoskeletons have been developed that superpose predefined trajectories to the user's joint motions and provide the level of assistance necessary to complete selected functional movements [2]. HMIs using model-free machine-learning approaches have also been proposed. These include pattern recognition and regression to map EMGs into joint kinematics.

However, one joint rotation can be generated via different EMG patterns. Therefore, mappings learned in one condition (such as light loads) may not generalize to novel conditions (heavy loads), leading to a lack of control robustness
[3]. An alternative approach is that of neuromusculoskeletal modeling, which explicitly characterizes each intermediate transformation between the EMG onset and joint-torque production by simulating the interplay among the nervous, muscular, and skeletal systems. We refer to this as a myoprocessor. Unlike a model-free machine-learning approach, a myoprocessor maps EMGs into forces that belong to the solution space of the human musculoskeletal system [4]. Therefore, decoded forces are always within physiological bounds, potentially enabling robust robot control across a broad range of conditions [5].

The design of powered orthoses has long been based on rigid exoskeletal technologies. This has the drawback of restricting body mobility, which induces misalignment with the user's joint [6] and leads to suboptimal ergonomics due to the device's excessive weight and size [7]. The introduction of soft robotic exosuits that employ flexible materials for transmitting forces to the human body has provided the possibility of addressing the limitations of rigid hardware [8]. Soft exosuits have the potential to effectively operate as an external layer superposed to biological muscles to support human-joint mechanics.

However, despite their potential benefits, exosuits still present limited control reliability [9] due to their highly nonlinear behavior, lower tracking accuracy, and reduced magnitude of assistance and control robustness when compared to rigid exoskeletons [10]. As a result, there is currently no effective framework for controlling soft wearable robots as a function of human motor intention [11].

We developed a new framework that combines a modelbased HMI with a soft, wearable arm exosuit to address the key limits of current HMIs and wearable robotics. This work assessed whether a myoprocessor based on an EMG-driven musculoskeletal model could be established and personalized to different individuals. We assessed the myoprocessor's accuracy in decoding elbow-joint torques from EMGs and translating the decoded torques into exosuit-control commands across a repertoire of dynamic joint rotations and load-lifting tasks. To the best of our knowledge, this is the first time that a subject-specific, EMG-driven musculoskeletal model is synthetized into an HMI for the online control of an upper-limb exosuit. Interfacing real-time myoprocessors with soft wearable robotics may lead to a new paradigm for achieving exosuit-control robustness as well as symbiotic human-exosuit interaction.

\section{Methods}

\section{Exosuit Design and Control Hardware}

A cable-driven elbow exosuit [12] (Figure 1) powered by a brushless electric motor (EC-i, 40-70 W, Maxon, Sachseln, Switzerland) was used to test the proposed controller. Sensing elements assembled in a load cell (LCM300, Futek, Irvine, California) secured on the distal anchor point of the exosuit fed the back-cable tension, while the elbow angular position was recorded by an absolute encoder (AS5047P, 
AMS, Premstaetten, Austria) aligned with the anatomical joint. To detect muscular bioelectric activity, a multichannel EMG system (Bagnoli Desktop, Delsys, Natick, Massachusetts) recorded the three muscles that contribute to flex and extend the elbow joint: namely, the long head of the biceps (BIC), long head of the triceps (TRI), and brachioradialis (BRD). The electrodes were placed according to the Surface EMG for Noninvasive Assessment of Muscles (SENIAM) guidelines [13]. All signals from the exosuit and EMG workstation were captured by an NI 6025E dataacquisition board (National Instruments, Austin, Texas) at a sampling frequency of $1 \mathrm{kHz}$, while an inner control loop for the exosuit actuation (Figure 2) ran at $10 \mathrm{kHz}$ on a dedicated motor controller (EPOS2 50/5, Maxon). The control architecture was arranged as two separate modules running concurrently. Real-time, low-level control of the exosuit and data logging were implemented as a MATLAB/ Simulink application. The exosuit high-level control was based on an EMG-driven musculoskeletal model implemented in $\mathrm{C}++[5]$.

\section{Real-Time Control Framework}

We developed a real-time framework composed of a highlevel controller (the myoprocessor) that estimated the joint torque from EMG signals and a low-level controller that provided the necessary assistance.

\section{Myoprocessor (High-Level Controller)}

Our real-time, EMG-driven modeling framework [3], [5] computed the elbow flexion-extension torque as a function of 1) three EMG channels and 2) the elbow flexion-extension joint angle from the exosuit encoder. The model included four components (Figure 2):

1) The activation-dynamics component [Figure 2(a)] converted the three input EMGs into muscle activations via a second-order muscle-twitch model and nonlinear transfer function. The resulting muscle activations were mapped to seven muscle-tendon units (MTUs). The activation derived from the BIC-EMG channel controlled the longand short-head MTUs of the BIC. Activation from the TRI-EMG channel controlled the long, lateral, and medialhead MTUs of the TRI. Activation from the BRD-EMG controlled the BRD and brachialis MTUs [3].

2) The MTU-kinematics component [Figure 2(a)] synthesized the 3D musculoskeletal geometry of the human arm [14] into a set of multidimensional, cubic B-splines.

3) The MTU-dynamics component [Figure 2(a)] solved for the muscle and tendon force using a Hill-type muscle model, as described in [3] and [5].

4) The torque-computation component projected the forces from the MTU dynamics onto the elbow joint using the moments arm from the MTU kinematics. The EMG-driven modeling framework was then connected to the exosuit's low-level controller via the user datagram protocol (UDP) to compute the assistance provided by the tendondriven transmission.

\section{Velocity and Torque Loops (Low-Level Controller)}

The elbow torque estimated via the myoprocessor was used as a reference signal for the exosuit's low-level admittance controller. The admittance controller was comprised of outer torque and inner velocity loops. The torque loop [Figure 2(b)] compared the reference torque, $\tau_{m}$, with the interaction torque, $\tau_{i}$. The interaction torque was estimated from a load cell that recorded the cable tension $(f)$ and transformed into elbow torque via the force/torque mapping [Figure 2(b)] [15].

Assuming that the anchor point was fixed with respect to the user's arm and neglecting the deformations of the fabric and the soft tissues, the interaction torque, $\tau_{i}$, was given by

$$
\tau_{i}=J\left(\phi_{e}\right)^{T} f
$$

where $J\left(\phi_{e}\right)$ was the cable's moment arm with respect to the elbow joint. This quantity was a function of the elbow angle, $\phi_{e}$, and defined as

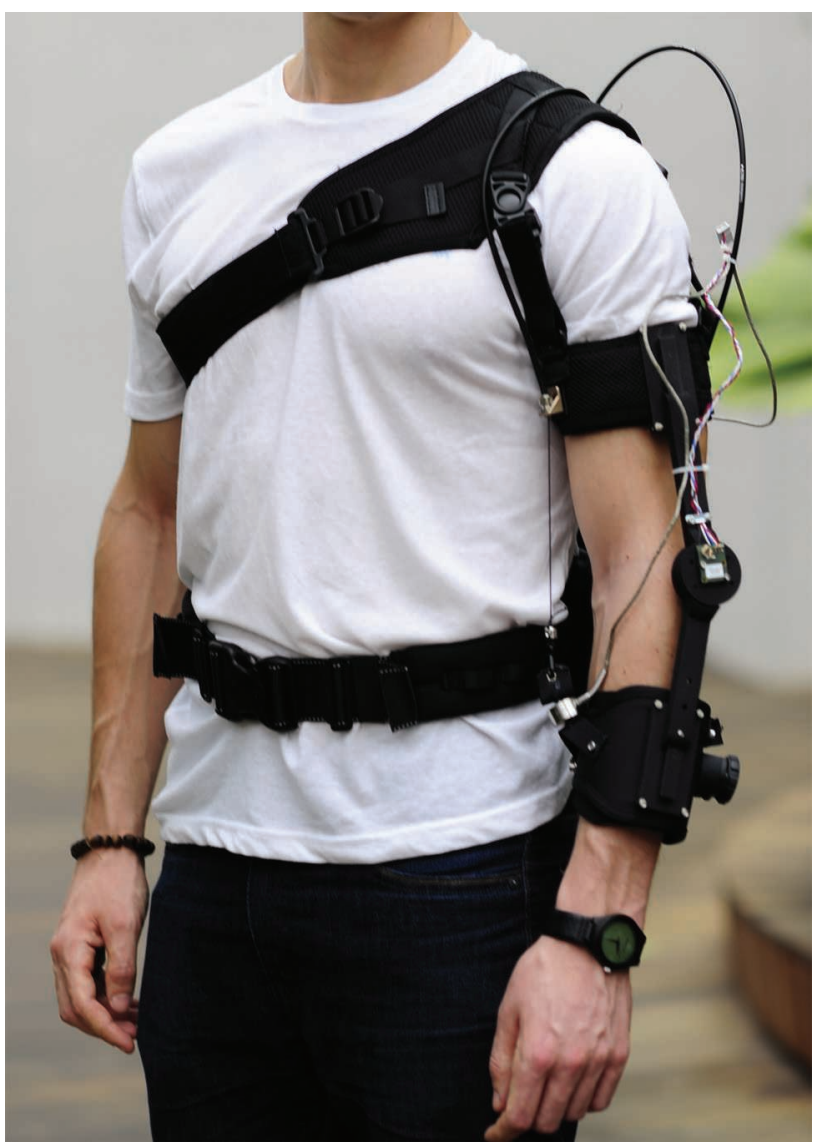

Figure 1. The exosuit device. The exosuit for elbow-joint assistance is composed of three fabric straps: one around the forearm (distal anchor point), one around the arm (proximal anchor point), and a shoulder harness connected to the arm strap via adjustable webbing bands. Buckles, Velcro straps, and a Boa lacing system enable the suit to be tightened. A pair of Bowden cables is attached to the front and back of the arm strap and transmits power from an actuation unit to the anchor points. The assistance is provided by recovering the front cable during elbow flexion. During elbow extension, the motor releases the cable. 


$$
J\left(\phi_{e}\right)=\frac{\partial h_{f}}{\partial \phi_{e}},
$$

where $h_{f}\left(\phi_{e}\right)$ was the cable-displacement function, which was described by the following model [see Figure 2(b) for details]:

$$
h_{f}\left(\phi_{e}\right)=2 \sqrt{a^{2}+b^{2}} \cos \left(\tan ^{-1}\left(\frac{a}{b}\right)+\frac{\phi_{e}}{2}\right)-2 b,
$$

where $a$ and $b$ were, respectively, the forearm's half width and the distance between the elbow's center of rotation and the anchor point.

The resulting torque error, $\tau_{r}=\tau_{m}-\tau_{i}$, was transformed into a desired angular velocity, $\omega_{r}$, through a proportionalintegral-differential (PID)-like admittance ("Target Admittance" in Figure 2) of the form

$$
Y(s)=\frac{\omega_{r}}{\tau_{r}}=P+\frac{I}{s}+D s,
$$

where the $P, I$, and $D$ gains shaped the dynamic response of the exosuit to follow the target, $\tau_{m}$, from the myoprocessor. The control gains were tuned to preserve stability, assuring a prompt response comparable with the requirements of the proposed tasks and minimizing the tracking error, $\tau_{r}$ [16]. They were tuned on a single subject prior to performing the experiments and left unchanged. Finally, the velocity loop [Figure 2(c)] was responsible for compensating for the intrinsic, unwanted dynamics of the exosuit device (backlash, static, and dynamic friction).

\section{Experiment Protocol}

The study involved six subjects: two females and four males, aged $26 \pm 2.4$ years [mean \pm standard deviation (SD)], with a body weight of $78.3 \pm 12 \mathrm{~kg}$, and a height of $1.78 \pm 0.08 \mathrm{~m}$. All participants had no evidence or known history of skeletal and neurological diseases, and they exhibited a normal joint range of motion and muscle strength. All experimental procedures were carried out in accordance with the Declaration of Helsinki on research involving human subjects and approved by the ethical committee of the University of Twente, Enschede, The Netherlands. All subjects provided their explicit written consent to participate in the study. To test the performance of the controller, we designed an experiment in which the subjects, wearing the exosuit, were requested to track a reference elbow trajectory, which was visually presented on a screen (see Figure 3).

The tracking task was performed under conditions where the exosuit was powered and unpowered. In the unpowered condition, the exosuit cable was slack, and the motor turned off. This enabled the replication of an unassisted scenario comparable to not wearing the device. The tracking task consisted of a single sequence of minimum-jerk trajectories, replicating features of physiological movements [17] in speed and amplitude.

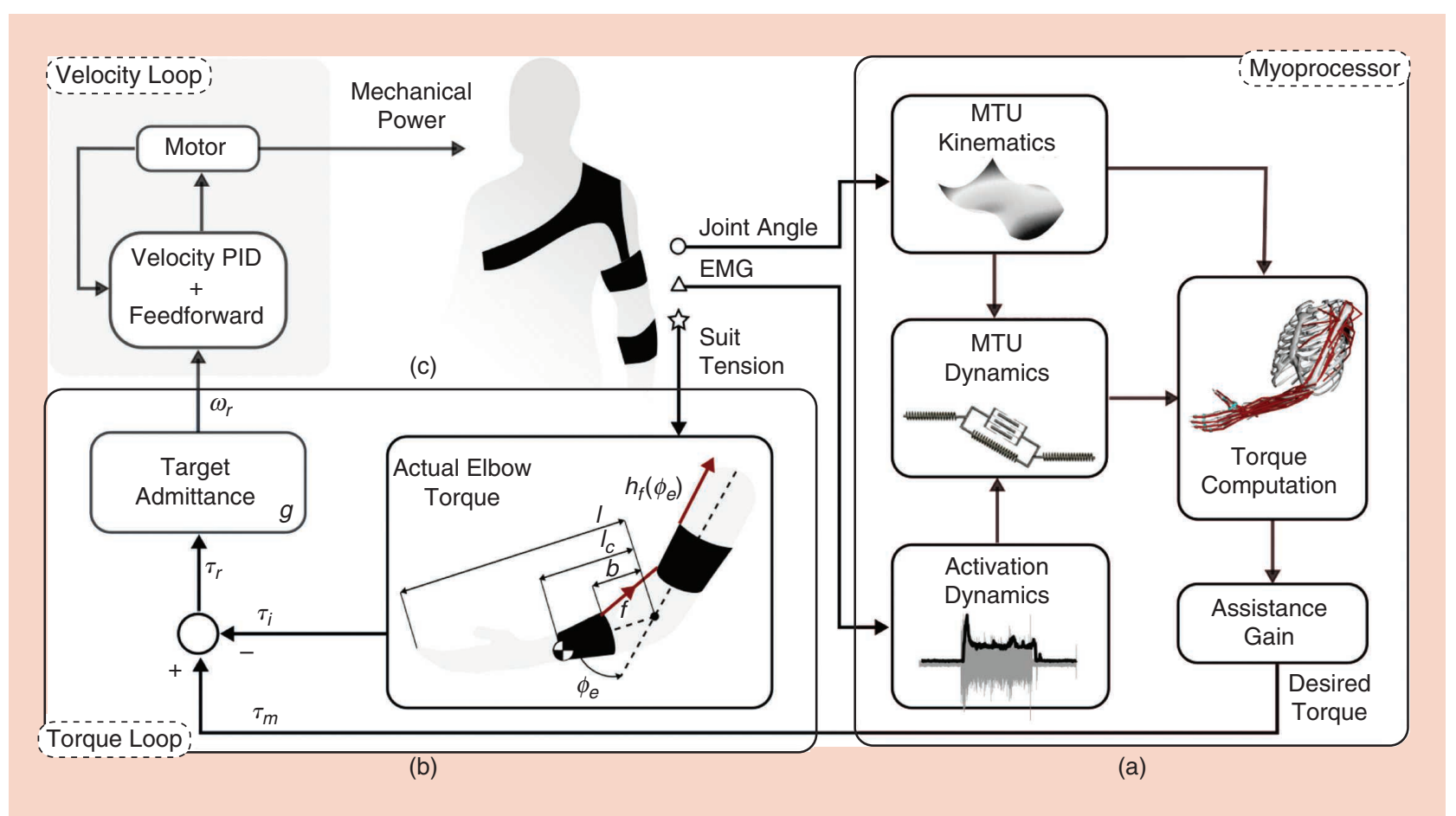

Figure 2. The exosuit control scheme. The (a) myoprocessor estimates physiologically accurate torque profiles $\left(\tau_{m}\right)$ equal to the torque generated by the muscles spanning the elbow joint. This is tracked by (b) an admittance controller consisting of an outer torque loop and (c) inner velocity loop. The myoprocessor combines geometric and dynamic properties of MTUs to estimate the net elbow flexion-extension torque as a function of muscle activation derived from surface EMG electrodes. The myoprocessor-estimated torque is compared to the torque $\left(\tau_{i}\right)$ delivered by the robot to the human joint. The error torque $\left(\tau_{r}=\tau_{m}-\tau_{i}\right)$ is then converted to a motor velocity, $\omega_{r}$, delivering assistive power to the suit's wearer. 
- Speed: We selected 35 and $70 \%$, which, respectively, corresponded to 27 and $55 \%$ of the average speed observed during the activities of daily living [18].

- Amplitude: Three movement amplitudes were tested, 30, 60 , and $80^{\circ}$ and each was repeated four times in a random order for a total of 12 movements per trial.

Similar to [12], the sequence was repeated for different load conditions. The load was placed on each subject's forearm through a specific support to avoid wrist flexion/extension and muscular activation at the level of the BRD nerve. There were three load conditions: $0 \mathrm{~kg}$ (unloaded), $1 \mathrm{~kg}$, and $2 \mathrm{~kg}$. We began the experiment with the unpowered condition for all subjects and randomized the load and velocity conditions. During the unpowered condition, another workstation ran the algorithm to calibrate the myoprocessor module by using the data collected during dynamic calibration. To avoid fatigue, the participants rested for $10 \mathrm{~min}$ between conditions.

\section{Myoprocessor Calibration}

Before running the experiment using the controller shown in Figure 2, each subject performed a calibration task. This enabled the musculoskeletal model of the myoprocessor to be tuned for individual anthropometric features. A 3D motioncapture system (Visualeyez II, Phoenix Technologies, Vancouver, Canada) recorded at $100 \mathrm{~Hz}$ the trajectories of six markers, which were placed on anatomical landmarks: the third metacarpus, lateral wrist, medial wrist, lateral elbow, medial elbow, and acromion. The calibration procedure consisted of three phases: 1) static-pose acquisition, 2) maximum voluntary contraction (MVC) trials, and 3) dynamic calibration. During the static pose, we recorded the $3 \mathrm{D}$ location of the reflective markers by means of motion capture. For each subject, the open source software OpenSim was used to linearly scale a generic musculoskeletal model [14] to match the individual's arm anthropometry. During the MVC trials, a series of isometric contractions for each muscle group was performed to extract the EMG peaks, which were used for the EMG-signal normalization.

During the dynamic calibration, the subjects were requested to follow reference trajectories (visual feedback on a screen) by flexing and extending their elbow at different speeds. The recorded EMG signals, joint angles, and reference torques were used to calibrate the myoprocessor; the joint moments were extracted using the OpenSim inverse-dynamics tool following the procedures described in [5]. The calibration process estimated the values of the internal model parameters that minimized the normalized error between the myoprocessor torque estimates and reference torque profiles. The tuned parameters included the optimal fiber length, tendon-slack length, maximal isometric force, and EMG-to-activation filtering coefficients [5].

\section{Data Analysis}

The raw EMG signals were processed online using a $50-\mathrm{Hz}$ notch filter, high-pass filtering $(35 \mathrm{~Hz})$, full-wave rectification, and low-pass filtering (4-Hz, second-order Butterworth filter) and normalized with respect to the individual MVC levels recorded during the calibration procedure. To test the subjects' performance across the different experimental conditions, a set of indicators was evaluated.

- Tracking accuracy: We computed the coefficient of determination, $r^{2}$, and the root-mean-square error (RMSE) between the reference and measured elbow trajectories performed by the subjects under the different conditions. The two metrics were also used to quantify the ability of the myoprocessor to estimate the elbow-joint torque through comparison with the OpenSim inversedynamics tool.

- Time delay: To assess the time lag between the EMG-signal onset and exosuit motor-drive engagement, we calculated the time window between the acquisition of the EMG signals and torque loop output, $\omega_{r}$. We used two approaches: offline and online. In the offline method, we simulated 140 EMG pulses and collected the output from the target admittance [Figure 2(b)], which drove the exosuit actuation. The online method estimated the onset time during the subjects' performance. We computed the norm of the derivative of the EMG linear envelopes and reference elbow angular velocity. In both signals, we estimated the onset as the time instant at which the signal was greater than $10 \%$ of its peak magnitude. We then extracted the time lag as the difference between the onset time of the velocity and muscle, with the BIC typically activated first.

- Muscular activity: We evaluated the RMS of the EMG signals and muscle forces, estimated from the myoprocessor, under all of the experimental conditions.

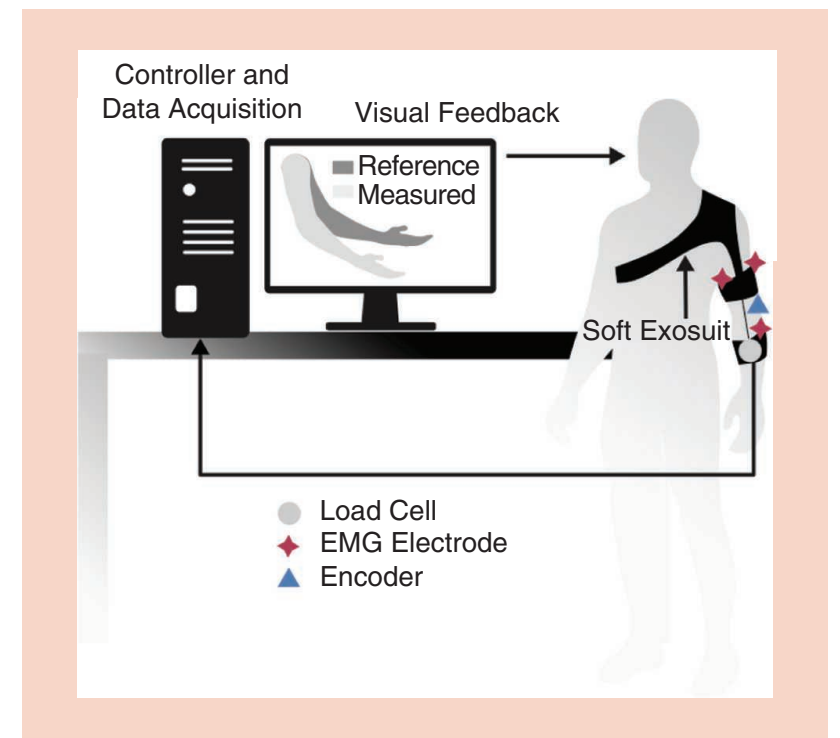

Figure 3. The tracking task to test the controller's performance. We instructed the subjects to follow a reference trajectory displayed on a screen in the form of a moving elbow; the position of their own arm was superimposed to provide visual feedback. The setup was the same for the powered and unpowered conditions. 


\section{Statistical Analysis}

Whenever appropriate, we assessed the normality of the statistical distribution using the Kolmogorov-Smirnov test and the sphericity condition for a repeated-measures analysis of variance (ANOVA) with the Mauchly test. All metrics resulted as normally distributed. When the sphericity condition was violated, we applied the Greenhouse-Geisser correction. The significance of the muscular-activation differences was inferred for different conditions using performance indicators (\%MVC and muscle forces) across the two main setups (the powered versus unpowered exosuit) and load conditions (unloaded versus $1 \mathrm{~kg}$ versus $2 \mathrm{~kg}$ ).

For the kinematic analysis, we considered the average value of the movement amplitude and velocity. A repeatedmeasures ANOVA within several subject factors was performed: powered versus unpowered, three loading conditions (unloaded, $1 \mathrm{~kg}$, and $2 \mathrm{~kg}$ ), and their mutual interaction. The significance level was set at $p<0.05$. For the ANOVA, we also reported the notation $F(n, d)$, where $n$ are the degrees of freedom (DoF) of the numerator (that is, the powered/unpowered condition) and $\mathrm{d}$ is the denominator (that is, the subjects).

\section{Results}

\section{Myoprocessor Reliably Estimated Elbow-Joint Torque}

Figure 4(a) displays the myoprocessor-estimated torque under the unpowered condition and zero load as well as the reference torque obtained by inverse dynamics. The coefficient of determination depicted in Figure 4(b) was calculated for each subject by averaging all of the movement amplitudes $\left(30,60\right.$, and $\left.80^{\circ}\right)$ and velocities (35 and $70 \%$ s).

For all subjects and across all trials, the coefficient of determination, $r^{2}$, was higher than 0.82 , with a mean value of $r^{2}=0.87 \pm 0.04$ (mean $\pm \mathrm{SD}$ ). The RMSE was lower than $0.02 \mathrm{~N} \cdot \mathrm{m} / \mathrm{kg}$, and the mean value was $0.0146 \pm 0.0051 \mathrm{~N} \cdot \mathrm{m} /$ $\mathrm{kg}($ mean $\pm \mathrm{SD})$.

\section{Exosuit Responsiveness}

The shorter the latency between the EMG onset and electromechanical actuation, the more transparent the exosuit was perceived to be by the wearer. This may be due to optimal synchronization between the muscle contraction and exosuit actuation. Figure $4(\mathrm{c})$ shows the distribution of the

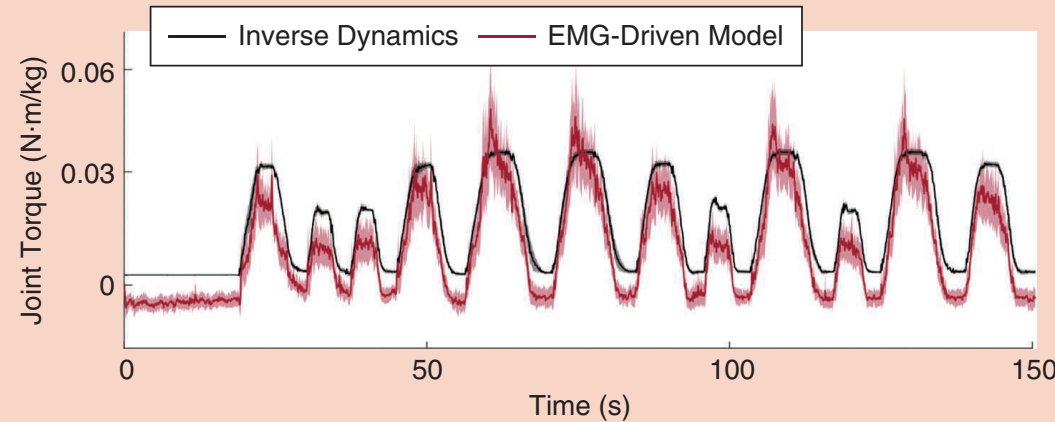

(a)

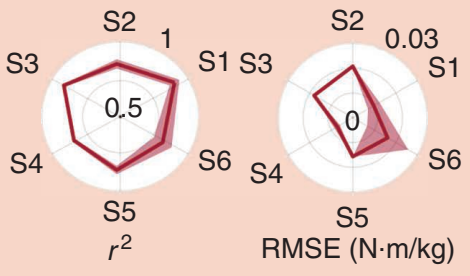

(b)

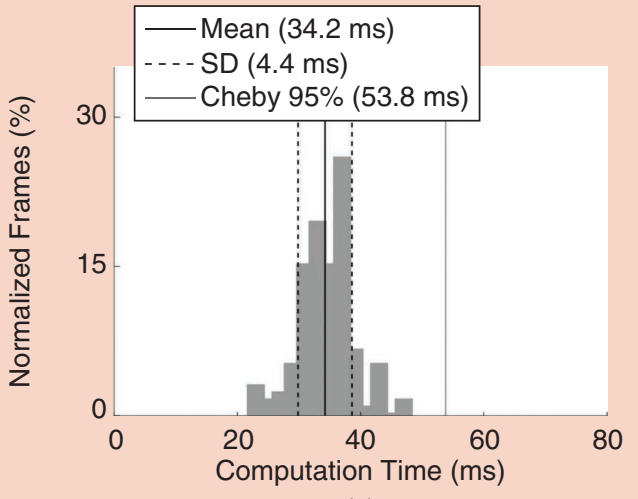

(c)

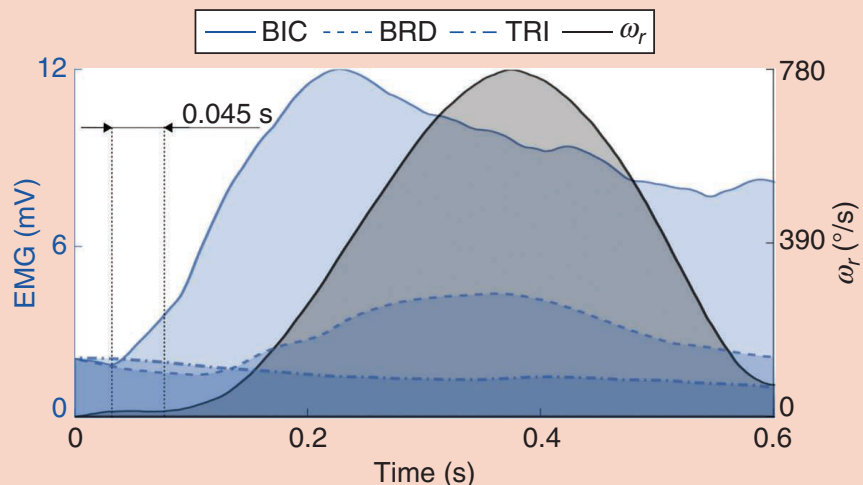

(d)

Figure 4. The accuracy and latency of the model-based myoprocessor. (a) The EMG-driven model-estimated torque, averaged across all of the subjects (red line, mean \pm SD), compared to the inverse dynamic torque (reference value, black line, mean \pm SD) during the tracking task. The torques were normalized using the mass of each subject. (b) The coefficient of determination, $r^{2}$, and the RMSE between the two torques for each subject, averaged across repetitions, velocities, and loads. (c) The distribution of the latencies between the EMG input and motor speed, including the average latency (mean \pm SD) and maximal expected error within the 95th percentile of the data. (d) A typical delay between the activation of the BIC and the target admittance output, $\omega_{r}$, [see Figure 2(b)] during a trial. The controller latency is within the time range of the electromechanical delay of the upper limbs' natural muscle fibers (55.5 ms). Cheby: Chebyshev's theorem. 
estimated latencies, including the mean latency and SD across all simulation frames (140 spike frames). Using Chebyshev's theorem, we estimated the maximal expected latency with $95 \%$ confidence. We found that in $95 \%$ of the cases the onset of the controller response occurred within $53.8 \mathrm{~ms}$, which is comparable to the physiological upperlimb electromechanical delay, i.e., $55.5 \mathrm{~ms}$, according to [19]. Figure 4(d) exemplifies a typical delay of $45 \mathrm{~ms}$ between the onset of the BIC and target admittance output, $\omega_{r}$, during a tracking movement.

\section{Assisted Elbow Motion}

Figure 5(a) shows that the reference-trajectory tracking accuracy was not affected by the exosuit assistance under the unpowered condition and in the presence of loads. The average tracking accuracy, measured using the coefficient of determination, $r^{2}$, during the unloaded trial $(0 \mathrm{~kg})$, for the unpowered condition was $0.94 \pm 0.03$, while for the powered condition it was $0.90 \pm 0.03$ (mean $\pm \mathrm{SD}$ ). We found similar values for the maximally loaded setup $(2 \mathrm{~kg})$, with the average $r^{2}$ being $0.94 \pm 0.03$ for the unpowered and $0.90 \pm 0.02$ for the powered conditions, respectively. Regarding the RMSE, we found $10.3^{\circ} \pm 2.3^{\circ}$ for the unpowered condition and $10.7^{\circ} \pm 2.5^{\circ}$ for the powered condition during the unloaded trial. In the maximally loaded setup ( $2 \mathrm{~kg})$, we found $9.0^{\circ} \pm 2.2^{\circ}$ during the unpowered condition and $11^{\circ} \pm$ $2.4^{\circ}$ during the powered condition. A Wilcoxon signed-rank test between the two aforementioned analyses confirmed that wearing the exosuit did not significantly reduce the ability to track a reference trajectory $(p=0.16)$ in terms of the delay when we looked at the $r^{2}$, but we found a significative difference in the RMSE value $(p=0.0034)$.

\section{Exosuit Assistance Reduced Muscular Activity}

A significant difference was observed in muscular activation across conditions. In particular, Figure 6 shows EMG traces for a representative subject performing the tracking movement during the unloaded condition [Figure 6(a)] and with 1-2 kg loads [Figure 6(b) and (c), respectively], with and without exosuit assistance. For the unloaded condition, the difference between the EMG waveforms was negligible, indicating that the controller did not feel the presence of the exosuit, assuring an almost complete mechanical transparency for the device. When movements were performed with a load, the EMG amplitude, with the exosuit assistance, was substantially lower, proving that the myoprocessor accurately estimated the elbow torque from the muscular activity and that, consequently, it delivered assistance to the subjects against gravity. Analysis of the muscular envelopes revealed that, in all muscles and powered conditions, the EMG activity decreased significantly with respect to the unpowered conditions. In particular, the BIC muscle displayed a significant decrease $[\mathrm{F}(1,5)=35.9, p=0.0018]$ as did the $\mathrm{BRD}[\mathrm{F}(1,5)=$ $12.6, p=0.016]$ and TRI $[\mathrm{F}(1,5)=9.8, p=0.026]$.

Figure 7(a) shows the trend of the EMG RMS, expressed as a percentage of the MVC, for two elbow velocities (minimum and maximum), with increasing loads and powered versus unpowered conditions. During the powered conditions, the activity of the BIC increased less than $2 \%$ between 1- and 2-kg loads. With regard to the muscle forces estimated by the myoprocessor [Figure 7(b)], we observed similar results, where the $\mathrm{BIC}[\mathrm{F}(1,5)=12.1, p=0.018], \mathrm{BRD}$ $[\mathrm{F}(1,5)=20.0, p=0.0066]$, and TRI $[\mathrm{F}(1,5)=14.6, p=0.012]$ presented a significant reduction under all powered conditions. Also, in this analysis, the BIC, which was the muscle

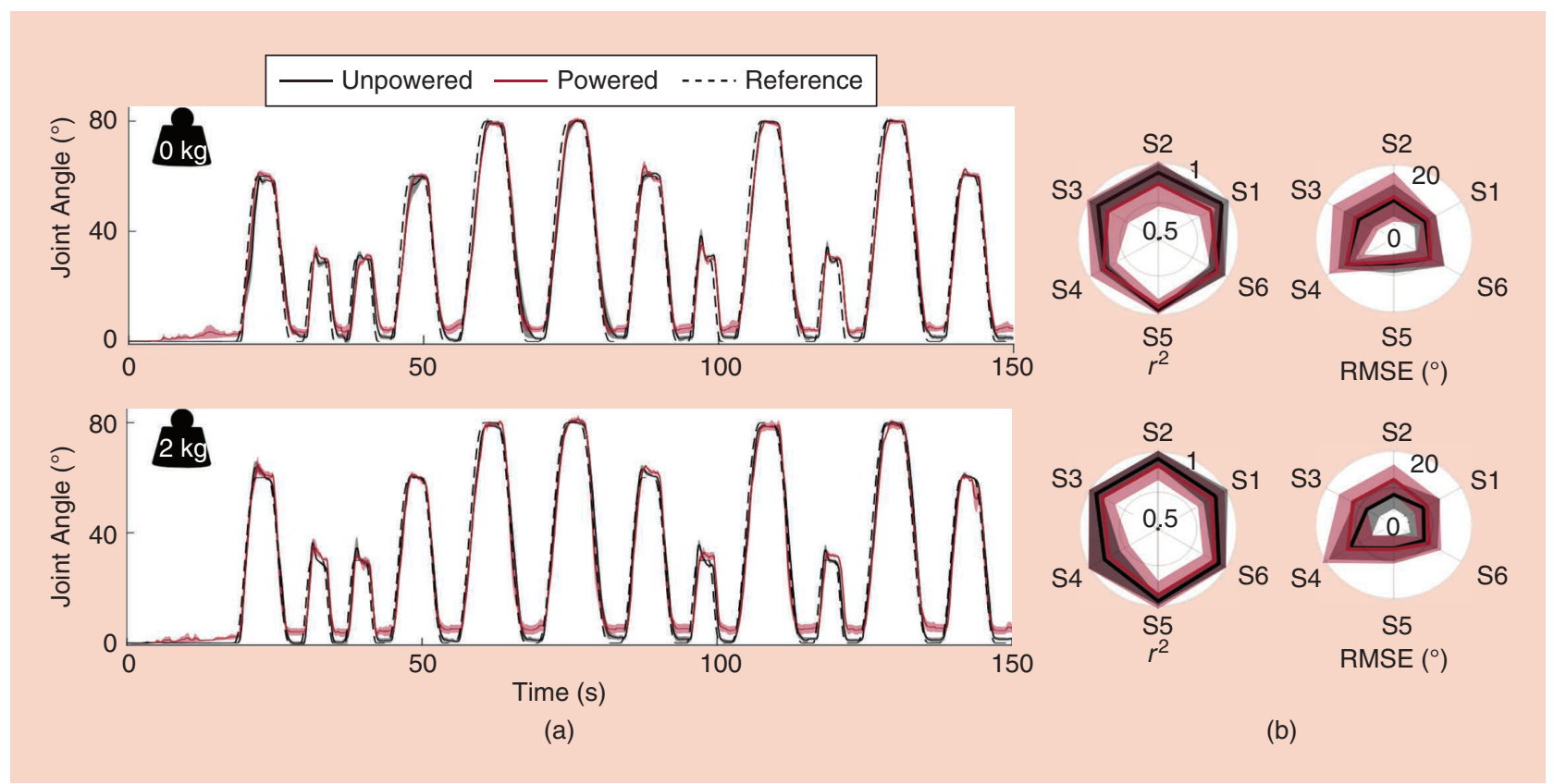

Figure 5. The human-tracking accuracy. (a) The tracking accuracy averaged across all of the subjects (mean \pm SD) for the (top) unloaded condition and (bottom) 2-kg condition. (b) The coefficient of determination, $r^{2}$, and the RMSE for each subject, computed on the (top) unloaded trial and (bottom) 2-kg trial. 
predominantly working against gravity, was found to increase by fewer than $5 \mathrm{~N}$ between 1- and 2-kg loads.

\section{Discussion}

We developed a human-machine interface that combined, for the first time, a realistic, subject-specific, real-time, EMG-driven myoprocessor with an upper-limb, active, soft exosuit. The proposed myoprocessor used the EMGs and kinematic data to estimate the net muscle-generated elbow flexion-extension torque, which was used to determine the assistance provided by the exosuit. That is, the exosuit added a fraction of the estimated elbow torque to support a range of movements. A distinctive aspect of this control scheme is that the myoprocessor directly incorporated changes in arm-plus-exosuit dynamics and task requirements (for example, the presence of additional loads and different speeds), with no need for additional recalibration and low-level controller retuning. In this way, the exosuit effectively operated as an extension of the human musculoskeletal system, dynamically adapting to different external mechanical loads.

We assessed the performance of the controller during tracking tasks (a sequence of arm flexion and extensions in the sagittal plane) carried out at different speeds and included lifting different loads. The results showed that the model-based estimates of the elbow torques matched the reference torque values well across all of the tested kinematic and load conditions [Figure 4(a)], thereby validating the model-estimation accuracy. The results also showed that the exosuit tracked the EMG-decoded elbow torques with high accuracy, thereby mimicking human musculoskeletal forces.
These are necessary conditions for actively supporting elbow rotations while not inducing unwanted motions and affecting natural movements, as usually happens with a rigid exoskeleton. In this context, the results from the human-tracking tests confirmed that during powered tasks the accuracy did not deteriorate with respect to the unpowered tasks across all subjects and trials (Figure 5). When compared with a previously presented gravity-compensation controller [12], our proposed solution displayed an improved tracking accuracy: the mean $r^{2}$ with gravitycompensator assistance was $0.8 \pm 0.06$, while our HMI was $0.9 \pm 0.04$. We found a significant difference in the RMS tracking-error variations $(p=0.034)$. However, the difference between the powered and unpowered conditions was always fewer than $2^{\circ}$. Importantly, the gravitycompensator controller [15] also needed PID-parameters tuning for each subject, which made it hard to provide adaptive assistance, depending on the external dynamic conditions. The PID module specifies the exosuit behavior at the human-machine physical interface, and the parameters need to be set to provide stability within the myoprocessor range of outputs and prevent saturation of the actuators. Since the myoprocessor output torque had similar magnitudes for all subjects, we tuned the PID module on the basis of one subject only. This procedure also reduced the controller set-up time and enabled us to focus on the myoprocessor's contribution to the system's overall performance. However, adapting the PID gain to individual subjects may further increase the control performance. Future studies will address a systematic analysis of how generic-versus-subject-specific PID gains affect the control performance.

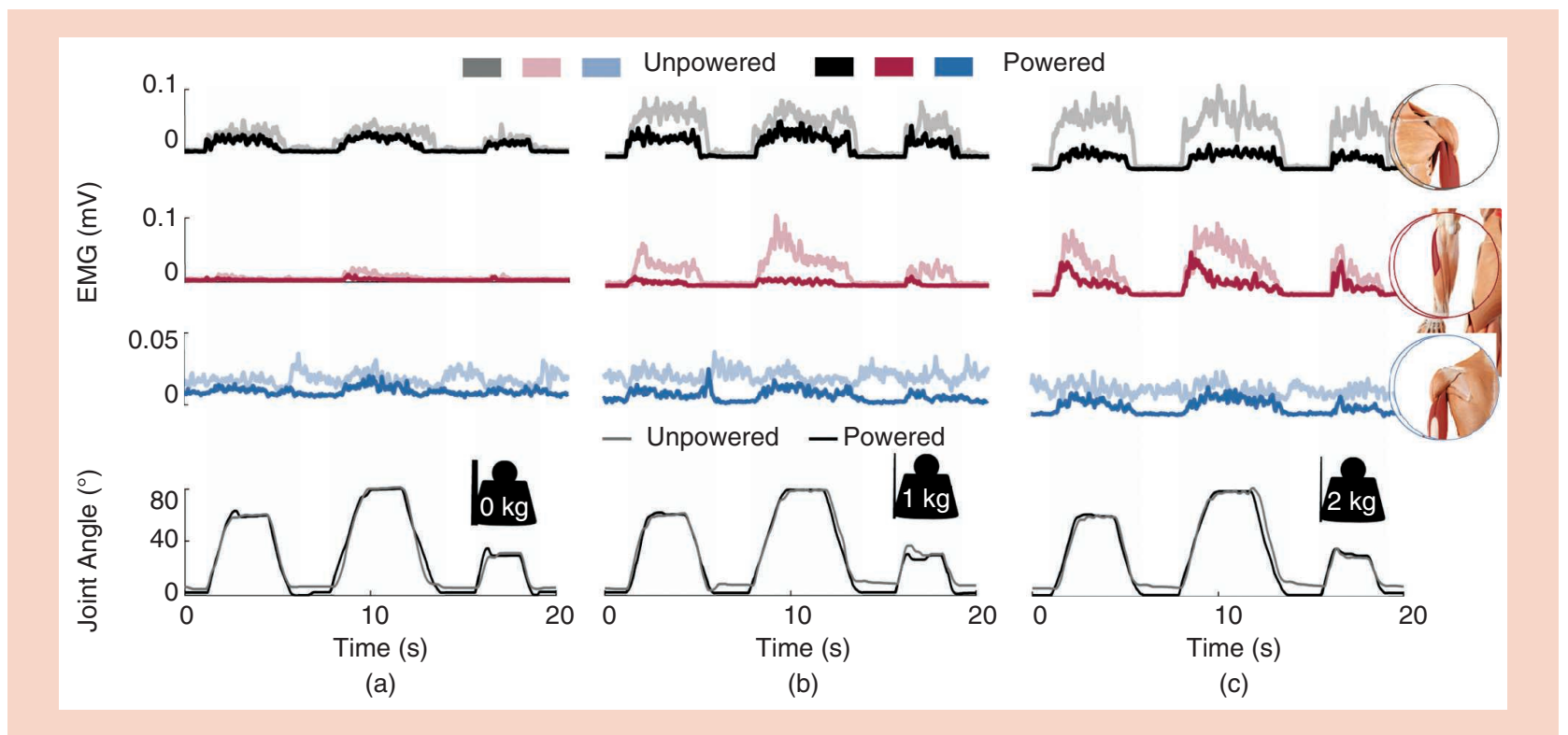

Figure 6. The muscle activation and tracking showing the EMG linear envelopes of a representative subject who performed three consecutive tasks at the highest velocity, with a (a) 0-kg load, (b) 1-kg load, and (c) 2-kg load under the powered and unpowered conditions. During the powered condition, the exosuit reduced the BIC activity by 35.6, 55.1, and 73\%; the BRD activity by 49.5, 77, and 58.4\%; and the TRI activity by 35.6, 59.7, and 51.9\% for the 0-, 1-, and 2-kg loads, respectively. 
Our results suggest that the exosuit's response to EMGs may be faster than HMIs based on human-exosuit interaction forces (Figure 5). EMG-controlled exosuits can react to EMGs theoretically before physical joint force and motion are generated. In the case of the interaction force, detectable joint force and motion must occur before the exosuit assistance can be computed and delivered to the human, something that would limit the final applicability; according to this scheme, exosuits can provide assistance only after detectable movement is produced by the user. Importantly, our approach did not require any hardware modification with respect to the interaction-force-based controllers that we previously developed and tested on the same device [12]. We used the same configuration shown in [12] and integrated our HMI in the control loop. Furthermore, our results showed that the exosuit intervention reduced the effort in the major elbow flexors across increasing velocity and load conditions: this was reflected by a decrease in the EMG and mechanical-force magnitude in the transition from unpowered to powered conditions; that is, the muscle EMGs and forces across the 1 - and 2-kg conditions varied within similar absolute ranges (Figure 7). Our results also showed that the exosuit support preserved the muscle-force-generating capacity across multiple velocities and loads [Figure 7(b)]. This condition was observed, in particular, on the BIC, the main elbow-flexor muscle.

The exosuit assisted only in flexion and enabled unassisted extension along gravity by releasing its artificial tendon. Therefore, the muscles that predominantly contributed to control the exoskeleton during flexion were the BIC and brachialis. However, it was necessary to include the TRI in the

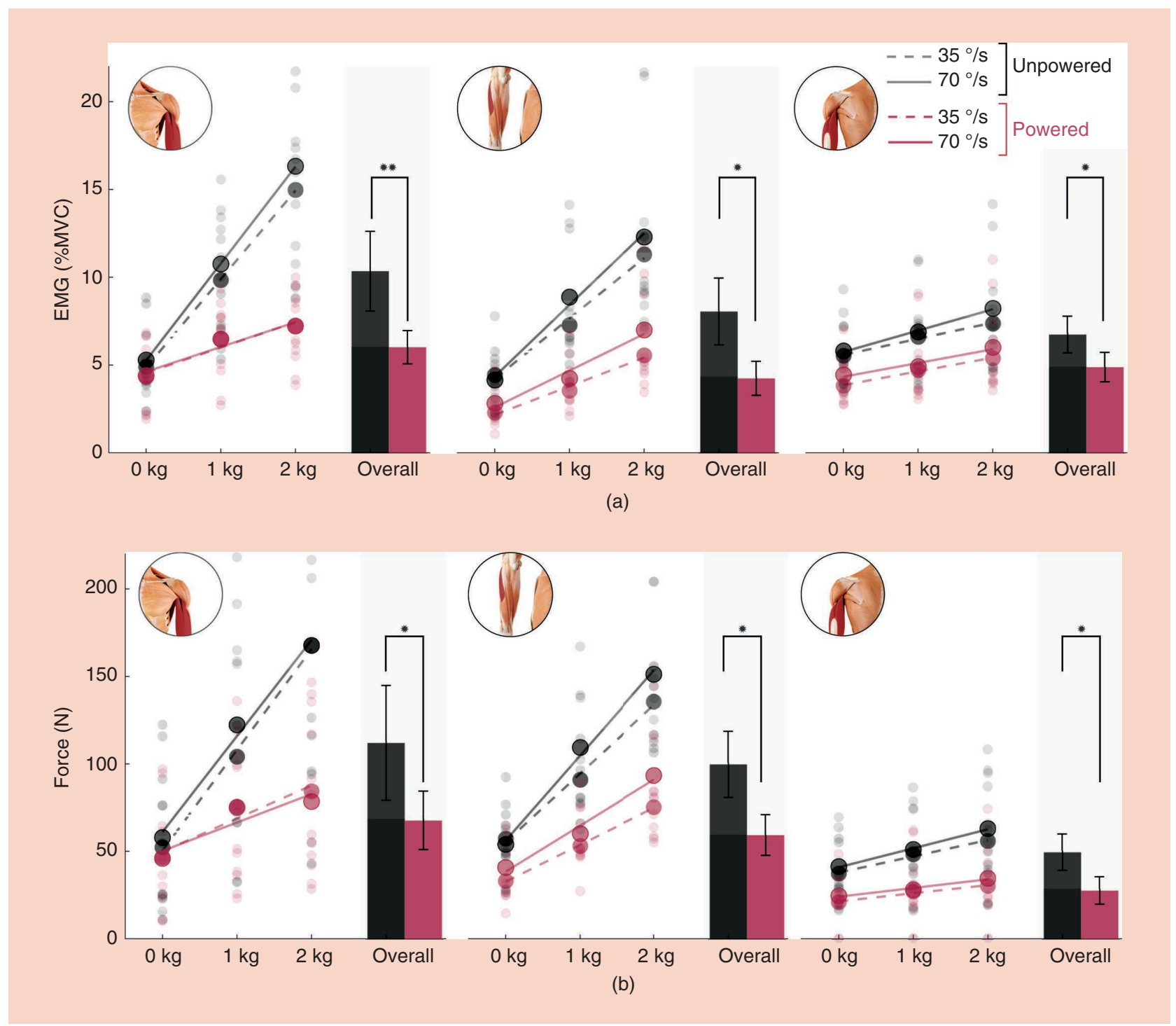

Figure 7. The EMG activity and estimated muscle force. The (a) EMG amplitude and (b) estimated muscle force. Translucent dots represent the values for each participant, and opaque dots denote the average across subjects. Lines indicate the trend across loads (a dashed line for $35 \%$ s and continuous line for $70 \%$ ). From left to right, we report the BIC, BRD, and TRI. The ** and * notations above the bar plots represent, respectively, highly significant $(p<0.01)$ and significant $(p<0.05)$ results. The EMG amplitude and force of all muscles were significantly lower in all powered conditions. 
myoprocessor module, for two reasons. The first concerned the elbow net-torque estimation to which the TRI contribute: In the hypothetical and extreme scenario where the subjects were able to exclusively contract the flexor muscles (leaving the TRI completely off), there would still be a passive force produced by the TRI due to muscle stretch. These forces could be on the order of several tens of newtons and, therefore, are not negligible. The correct estimation of these passive resistive forces would provide the best possible estimation of the net elbow torque. If we had neglected these passive torques, we could have had an over- or underestimation of the net elbow torque, thereby providing suboptimal assistance to the human.

The second reason regarded the exosuit control: the mechanical system was not back-drivable, so the TRI was used to release the artificial tendon during elbow extension, and its initial burst triggered the release of the motor and made the device transparent during elbow extension, as well. In this context, the reduction in the TRI muscle activity (Figure 7) was an unexpected result. It could have been caused by the participants having to follow a predefined trajectory displayed on a screen. This might have led to the descending motion to deviate from the purely concentric, which would imply the modulation of agonist and antagonist muscle to preserve accuracy (Figure 6).

In this context, our results showed that the BIC activity during the powered condition varied minimally across loads: this provided evidence that the proposed myoprocessor could make the worn device and external load transparent to the human operator during dynamic tasks. Since small discrepancies between the onset of the human limb movement and the motion of the parallel exosuit can significantly increase human muscle effort, the results demonstrated that our approach ensured a reliable synchronization that could directly exploit the electromechanical delay (EMD) for prompt device control. Our results showed that, across the recruited subjects, the EMD was sufficiently large (20-48 ms) (Figure 4) for decoding mechanical moments from the EMGs before the voluntary movement took place. Moreover, the EMD was sufficiently consistent to be considered a hard, real-time deadline for the exosuit control. Meeting this deadline within our proposed HMI assured that the exosuit would be actuated synchronously with the user's muscle mechanical function. This is an additional advantage with respect to current HMIs that actuate wearable robots solely on the basis of detecting externally measurable forces, which does not provide support until the operator has produced detectable dynamics at the interface with the machine [8].

Our study presented limitations. We explored a restricted range of movements; this was due to constraints in the exosuit actuation stage. The experiments involved only healthy subjects; our future work will involve neurologically impaired individuals. The device actuated a single DoF, so during calibration it was important to avoid movement that involved other joints. In this study we constrained the wrist to best test our approach. The BRD is a biarticular muscle that spans the wrist and elbow; the exosuit, however, was only designed for the elbow flexion-extension. We did not model any DoF at the wrist, and, therefore, we avoided motions there. Our upcoming work will focus on testing the control of multiple soft mechatronic DoF: for example, simultaneous control of the elbow and hand joints in exosuits.

Our experiments required subject-specific calibration. However, it is worth stressing that the calibration does not need to be performed every time one uses the device. In this context, there are two types of parameters in the myoprocessor module: anatomical and filtering. Once identified, anatomical parameters should not vary for a grown adult because they are linked to the anatomy of the subject. In a previous study [3] that used the same myoprocessor, the model calibration was always performed a number of days prior to the real-time prosthesis-control experiments. This provided evidence of the EMG-model framework's ability to retain subject-specific parameter consistency across time scales. On the other hand, filtering parameters (the MVC and EMG-to-activation coefficients) may be less stationary since they depend on EMG-skin impedance, fatigue, and other factors, but this quantification requires a stand-alone study, which is planned for the future.

In this study, we did not compare our HMI with different approaches, such as simpler direct-EMG controllers. In this context, the added complexity of our model is repaid by a twofold benefit: 1) the ability to capture and adapt to unpredictable and varying external dynamics, tested under the different load conditions (Figure 6) [7] and 2) a higher robustness against movement artefacts and noise in the EMG signals. A proportional controller, based only on processed EMG signals, could not capture all aspects related to the dynamics of the arm, since it ignored muscle geometry and EMG-to-activation conversion. The second advantage directly translates to a higher usability in real-world scenarios. In [3], we showed that the myoprocessor, relying on muscular geometry as well as activation levels, is robust against mechanically induced movement artefacts in the EMG signals, such as arm positions and cable movements. In future studies, we will directly compare the myoprocessor with other myoelectric control techniques (for example, proportional EMG control) by enlisting a larger number of subjects and conditions (after hardware improvements) to test the generalizability. We will also test the exosuit's control on subjects with neuromuscular conditions to assess the extent to which the model-based HMI can adapt to abnormal anatomies and affected muscular activation patterns [20]. Finally, the study explored only highly controlled elbow rotations and did not include functional real-life tasks. However, our approach demonstrated an adaptation to external loads that could be applied in unstructured environments. The robustness of the controller, combined with a symbiotic HMI, can provide cooperation between the user and exosuit. The next step will adapt the system to port this device in the real world.

In conclusion, we demonstrated the possibility of controlling exosuits via a real-time myoprocessor, enabling adaptive support under a diverse range of mechanical loads and kinematics during elbow motion. The integration of model-based 
HMIs with soft mechatronics has the potential to open new frontiers in highly ergonomic wearable assistive robots that will behave symbiotically with humans.

\section{Acknowledgements}

Michele Xiloyannis and Guillaume Durandau contributed equally to this work. Lorenzo Masia and and Massimo Sartori contributed equally to this work. This work was partly supported by the European Research Council Starting Grant INTERACT (803035).

\section{References}

[1] M. Sartori, D. G. Llyod, and D. Farina, "Neural data-driven musculoskeletal modeling for personalized neurorehabilitation technologies," IEEE Trans. Biomed. Eng., vol. 63, no. 5, pp. 879-893, May 2016. doi: 10.1109/TBME.2016.2538296.

[2] H. S. Lo and S. Q. Xie, "Exoskeleton robots for upper-limb rehabilitation: State of the art and future prospects," Med. Eng. Phys., vol. 34, no. 3, pp. 261-268, Apr. 2012. doi: 10.1016/j.medengphy.2011.10.004.

[3] M. Sartori, G. Durandau, S. Došen, and D. Farina, "Robust simultaneous myoelectric control of multiple degrees of freedom in wrist-hand prostheses by real-time neuromusculoskeletal modeling," J. Neural Eng., vol. 15, no. 6, p. 066026, Oct. 2018. doi: 10.1088/1741-2552/aae26b.

[4] E. E. Cavallaro, J. Rosen, J. C. Perry, and S. Burns, "Real-time myoprocessors for a neural controlled powered exoskeleton arm," IEEE Trans. Biomed. Eng., vol. 53, no. 11, pp. 2387-2396, Nov. 2006. doi: 10.1109/TBME.2006.880883.

[5] G. Durandau, D. Farina, and M. Sartori, "Robust real-time musculoskeletal modeling driven by electromyograms," IEEE Trans. Biomed. Eng., vol. 65, no. 3, pp. 556-564, Mar. 2018. doi: 10.1109/TBME.2017. 2704085.

[6] A. Schiele and F. C. Van Der Helm, "Kinematic design to improve ergonomics in human machine interaction," IEEE Trans. Neural Syst. Rehabil. Eng., vol. 14, no. 4, pp. 456-459, Dec. 2006. doi: 10.1109/ TNSRE.2006.881565.

[7] A. Frisoli, F. Rocchi, S. Marcheschi, A. Dettori, F. Salsedo, and M. Bergamasco, "A new force-feedback arm exoskeleton for haptic interaction in virtual environments," in Proc. 1st Joint Eurohaptics Conf. and Symp. Haptic Interfaces Virtual Environment and Teleoperator Systems. World Haptics Conf., 2005, pp. 195-201. doi: 10.1109/WHC.2005.15.

[8] A. T. Asbeck, S. M. De Rossi, I. Galiana, Y. Ding, and C. J. Walsh, "Stronger, smarter, softer: Next-generation wearable robots," IEEE Robot. Autom. Mag., vol. 21, no. 4, pp. 22-33, Dec. 2014. doi: 10.1109/ MRA.2014.2360283.

[9] Y. Ding et al., "Biomechanical and physiological evaluation of multi-joint assistance with soft exosuits," IEEE Trans. Neural Syst. Rehabil. Eng., vol. 25, no. 2, pp. 119-130, Feb. 2017. doi: 10.1109/TNSRE. 2016.2523250.

[10] D. Park and K.-J. Cho, "Development and evaluation of a soft wearable weight support device for reducing muscle fatigue on shoulder," PLoS One, vol. 12, no. 3, p. e0173730, Mar. 2017. doi: 10.1371/journal. pone.0173730.

[11] K. Anam and A. A. Al-Jumaily, "Active exoskeleton control systems: State of the art," Procedia Eng., vol. 41, pp. 988-994, 2012. doi: 10.1016/j. proeng.2012.07.273. [Online]. Available: http://www.sciencedirect.com/ science/article/pii/S1877705812026732
[12] M. Xiloyannis, D. Chiaradia, A. Frisoli, and L. Masia, "Physiological and kinematic effects of a soft exosuit on arm movements," J. Neuroeng. Rehabil., vol. 16, no. 1, p. 29, Feb. 2019. doi: 10.1186/s12984 -019-0495-y.

[13] H. J. Hermens, B. Freriks, C. Disselhorst-Klug, and G. Rau, "Development of recommendations for SEMG sensors and sensor placement procedures," J. Electromyogr. Kinesiol., vol. 10, no. 5, pp. 361-374, Oct. 2000. doi: 10.1016/S1050-6411(00)00027-4.

[14] K. R. Holzbaur, W. M. Murray, and S. L. Delp, "A model of the upper extremity for simulating musculoskeletal surgery and analyzing neuromuscular control," Ann. Biomed. Eng., vol. 33, no. 6, pp. 829-840, June 2005. doi: 10.1007/s10439-005-3320-7.

[15] D. Chiaradia, M. Xiloyannis, C. W. Antuvan, A. Frisoli, and L. Masia, "Design and embedded control of a soft elbow exosuit," in Proc. 2018 IEEE Int. Conf. Soft Robotics (RoboSoft), pp. 565-571. doi: 10.1109/ ROBOSOFT.2018.8405386.

[16] W. Yu, J. Rosen, and X. Li, "PID admittance control for an upper limb exoskeleton," in Proc. 2011 American Control Conf., pp. 1124-1129. doi: 10.1109/ACC.2011.5991147.

[17] T. Flash and N. Hogan, "The coordination of arm movements: An experimentally confirmed mathematical model," J. Neurosci., vol. 5, no. 7, pp. 1688-1703, July 1985. doi: 10.1523/JNEUROSCI.05-07-01688.1985.

[18] M. A. Buckley, A. Yardley, G. R. Johnson, and D. A. Cams, "Dynamics of the upper limb during performance of the tasks of everyday living: A review of the current knowledge base," J. Eng. Med., vol. 210, no. 4, pp. 241-247, Dec. 1996. doi: 10.1243/PIME_PROC_1996_210_420_02.

[19] R. W. Norman and P. V. Komi, "Electromechanical delay in skeletal muscle under normal movement conditions," Acta Physiol. Scand., vol. 106, no. 3, pp. 241-248, July 1979. doi: 10.1111/j.1748-1716.1979.tb06394.x. [20] G. Durandau et al., "Voluntary control of wearable robotic exoskeletons by patients with paresis via neuromechanical modeling," J. Neuroeng. Rehabil., vol. 16, no. 1, p. 91, July 2019. doi: 10.1186/s12984-019-0559-z.

Nicola Lotti, Universität Heidelberg, Germany, and Universitá degli studi di Genova, Genoa, Italy. Email: nicola.lotti@ziti .uni-heidelberg.de.

Michele Xiloyannis, ETH Zürich, Switzerland. Email: michele .xiloyannis@hest.ethz.ch.

Guillaume Durandau, University of Twente, Enschede, The Netherlands. Email: g.v.durandau@utwente.nl.

Elisa Galofaro, Universitá Degli Studi di Genova, Genoa, Italy. Email: elisa.galofaro@edu.unige.it.

Vittorio Sanguineti, Universitá Degli Studi di Genova, Genoa, Italy. Email: vittorio.sanguineti@unige.it.

Lorenzo Masia, Universität Heidelberg, Germany, and Southern Denmark University, Odense. Email: lorenzo.masia@ziti .uni-heidelberg.de.

Massimo Sartori, Universiteit Twente, Enschede, The Netherlands.Email: m.sartori@utwente.nl. 